Part of Journal of Research of the National Bureau of Standards, Volume 19, November 1937

\title{
EFFECT OF WAVE FORM UPON THE PERFORMANCE OF CURRENT TRANSFORMERS
}

\author{
By John H. Park
}

\section{ABSTRACT}

The effects of changing the wave form of the primary current from sinusoidal to peaked or flat top (20-percent third harmonic) upon the ratio and the phase angle of several current transformers and upon the performance of a Silsbee currenttransformer testing set were determined experimentally. Definitions of the ratio and the phase angle of a current transformer under nonsinusoidal conditions of current were derived in terms of the ratios of transformation and the shifts in phase angle of the fundamental and of the harmonic components of the primary current due to transformation.

\section{CONTENTS}

I. Introduction

II. Definitions of ratio and phase angle for nonsinusoidal conditions

1. Ratio

2. Phase anglé. 519

III. Apparatus and procedure. 523

IV. Results and conclusions

\section{INTRODUCTION}

Commercial measurements of electric power and energy, in circuits carrying alternating currents of large magnitude, are invariably made by the use of current transformers which supply, to the measuring instruments proper, secondary currents which are replicas on a reduced scale of the corresponding currents in the primary circuit. In important installations, the proportionality factor or "ratio" of the transformer is obtained by an experimental comparison between the transformer in question and a standard transformer of known ratio. This comparison is usually made in the testing laboratory of the utility company concerned, and the standard transformer in turn is tested at suitable intervals by some absolute method, usually in a standardizing laboratory, such as that of the National Bureau of Standards.

It is obvious that the accuracy of the final results would be affected if the conditions under which either the working or the standard transformer is operated were allowed to differ to a significant extent from the conditions under which either transformer was tested. With ordinary care such conditions as frequency and secondary burden can be readily reproduced with sufficient fidelity, and the effect of changes in these conditions decreases in general with an increase in the current to be measured, and hence with the commercial importance of the measurement. The variations in ratio and phase angle of a current transformer resulting from changes in the geometrical configuration 
of its primary circuit have been investigated and the results published. ${ }^{1}$ While these variations are negligible in multiturn transformers of low current rating, they tend to increase as the rated primary current increases and if accurate results are desired in the measurements of large alternating currents it becomes necessary to test the current transformer involved, with the same arrangement of primary circuit as will be used when the transformer is in operation.

The effect of variations in the wave form of the current being measured on the ratio and phase angle of a current transformer has received very little consideration since an investigation by Agnew ${ }^{2}$ from which he concluded that the effect was negligible for commercial wave forms. Agnew's investigation, however, was limited to transformers of low primary-current rating, and since the measurement of alternating currents of from 500 to 4,000 amperes has become quite common in recent years it was decided that an investigation of the effect of wave form upon the ratio and the phase angle of current transformers of this range was desirable.

\section{DEFINITIONS OF RATIO AND PHASE ANGLE FOR NONSINUSOIDAL CURRENTS}

The ratio and phase angle of a current transformer, as measured by a majority of the methods now in use, give the relation between the fundamental components of the primary and the secondary currents. Unless the current being measured is nearly sinusoidal, this relation may not be the most useful one obtainable. Therefore, it becomes necessary in this investigation to measure not only the ratio and the phase angle between the fundamental components of the primary and the secondary currents, but also the ratios and the phase angles between the harmonic components of the primary current and the corresponding harmonic components of the secondary current. In order to express the results of this work in terms of quantities which are really important in the use of the current transformer, definitions of the ratio and the phase angle of a current transformer were derived in terms of the measured quantities.

\section{RATIO}

In defining the ratio of a current transformer for use in measurements of nonsinusoidal currents it will be assumed that the current to be measured contains an indefinite number of odd harmonics and that its instantaneous value can be represented by the equation

$$
i_{1}=I_{m}\left[\sin \omega t+k_{3} \sin \left(3 \omega t+\lambda_{3}\right)+k_{5}\left(\sin 5 \omega t+\lambda_{5}\right)+\cdots\right]
$$

If $N c_{1}, N c_{3}, N c_{5}, \ldots$ denote the ratios in which the fundamental and the $3 \mathrm{~d}, 5 \mathrm{th}, \ldots$ harmonic components of the primary current are transformed and if $\beta_{1}, \beta_{3}, \beta_{5}, \ldots$ denote the corresponding phase angles, the instantaneous value of the secondary current of the transformer can be represented by

$$
i_{2}=\frac{I_{m}}{N}\left[\frac{1}{c_{1}} \sin \left(\omega t+\beta_{1}\right)+\frac{k_{3}}{c_{3}} \sin \left(3 \omega t+\lambda_{3}+\beta_{3}\right)+\frac{k_{5}}{c_{5}} \sin \left(5 \omega t+\lambda_{5}+\beta_{5}\right)+\cdots\right]
$$

1 John H. Park. Accuracy of high-range current transformers. J. Research NBS 14, 367 (1935) RP775.

${ }^{2} \boldsymbol{A}$ study of the current transformer with particular reference to iron losses. Bul. BS 7, 470 (1911) S164. 
The constant $N$ is the nominal ratio of the current transformer and the $c$ 's are ratio correction factors, i. e., the factors by which the nominal ratio must be multiplied to obtain the true ratios.

The usually accepted definition of the ratio of a current transformer is the ratio of the effective or rms value of primary current to the effective or rms value of secondary current. With this definition as a basis the ratio for nonsinusoidal currents is to be expressed in terms of the ratios of the fundamental and the harmonic components. From eq 1 and 2 the effective values of the primary current and the secondary current are

and

$$
I_{1}=\frac{I_{m}}{\sqrt{2}}\left(1+k_{3}{ }^{2}+k_{5}{ }^{2}+\cdots \cdot\right)^{\frac{1}{2}}
$$

$$
I_{2}=\frac{I_{m}}{N \sqrt{2}}\left(\frac{1}{c_{1}^{2}}+\frac{k_{3}{ }^{2}}{c_{3}{ }^{2}}+\frac{k_{5}{ }^{2}}{c_{5}^{2}}+\cdots\right)^{\frac{1}{2}}
$$

If the ratio of effective values is denoted by $N c_{e}$, where, as before, $N$ is the nominal ratio, and $c_{e}$ is the ratio correction factor,

$$
N c_{e}=\frac{I_{1}}{I_{2}}=N\left(\frac{1+k_{3}{ }^{2}+k_{5}{ }^{2}+\cdots}{\frac{1}{c_{1}{ }^{2}}+\frac{k_{3}{ }^{2}}{c_{3}{ }^{2}}+\frac{k_{5}{ }^{2}}{c_{5}{ }^{2}}+\cdots}\right)^{\frac{1}{2}}
$$

Performing the indicated division within the parenthesis, and neglecting terms such as $\left(1-\frac{c_{1}{ }^{2}}{c_{n}{ }^{2}}\right) k_{n}{ }^{4}$ in the fourth and higher powers of $k_{n}$

$$
c_{e}=c_{1}\left[1+\left(1-\frac{c_{1}^{2}}{c_{3}^{2}}\right) k_{3}{ }^{2}+\left(1-\frac{c_{1}{ }^{2}}{c_{5}^{2}}\right) k_{5}{ }^{2}+\cdots\right]^{\frac{1}{3}}
$$

It will be assumed here that $k_{n} \leqq 0.2$. Thus, the terms in $k^{2}$ will be quite small, and since each one of the $c$ 's is nearly unity, eq 6 becomes

$$
c_{e}=c_{1}\left[1+\left(c_{3}-c_{1}\right) k_{3}{ }^{2}+\left(c_{5}-c_{1}\right) k_{5}{ }^{2}+\ldots . .\right]
$$

Equation 7 is in convenient form for computing the ratio of the effective primary current to the effective secondary current from the ratios of transformation for the fundamental and the harmonic components. It may be noted that unless the ratios for the harmonic components are considerably different from the ratio for the fundamental, the ratio of the effective primary current to the effective secondary current is very nearly the same as the ratio of transformation for the fundamental component.

\section{PHASE ANGLE}

The phase angle of a current transformer for currents of sinusoidal wave form is customarily defined as the angle between the primarycurrent vector and the secondary-current vector reversed. This definition cannot be used for nonsinusoidal currents since they cannot be represented by single vectors. 
The phase angle of a current transformer is of importance only when power or energy is to be measured. For either of these measurements, with nonsinusoidal wave forms, a definition of phase angle must take account not only of the current but also of the load circuit and hence of the voltage across the load. If a primary current as represented by eq 1 is assumed to flow through a load circuit of resistance $R$ and inductance $L$, the instantaneous voltage across this circuit is

$$
\begin{aligned}
& e_{1}=I_{m}\left[\left(R^{2}+\omega^{2} L^{2}\right)^{\frac{1}{2}} \sin \left(\omega t+\tan ^{-1} \frac{\omega L}{R}\right)+\right. \\
& k_{3}\left(R^{2}+9 \omega^{2} L^{2}\right)^{\frac{1}{2}} \sin \left(3 \omega t+\lambda_{3}+\tan ^{-1} \frac{3 \omega L}{R}\right)+ \\
& \left.k_{5}\left(R^{2}+25 \omega^{2} L^{2}\right)^{\frac{1}{2}} \sin \left(5 \omega t+\lambda_{5}+\tan ^{-1} \frac{5 \omega L}{R}\right)+\ldots\right]
\end{aligned}
$$

The effective value of this voltage is

$$
E_{1}=\frac{I_{m}}{\sqrt{2}}\left[R^{2}+\omega^{2} L^{2}+k_{3}{ }^{2}\left(R^{2}+9 \omega^{2} L^{2}\right)+k_{5}{ }^{2}\left(R^{2}+25 \omega^{2} L^{2}\right)+\ldots\right]^{\frac{1}{2}}
$$

Since the current in this circuit is $i_{1}$ (see eq 1 ) and the voltage across it is $e_{1}$ (see eq 8), the apparent power is

$$
\begin{aligned}
& U_{1}=E_{1} I_{1}=\frac{I_{m}{ }^{2}}{2}\left\{[ 1 + k _ { 3 } { } ^ { 2 } + k _ { 5 } { } ^ { 2 } + \ldots . ] \left[R^{2}+\omega^{2} L^{2}\right.\right. \\
& \left.\left.+k_{3}{ }^{2}\left(R^{2}+9 \omega^{2} L^{2}\right)+k_{5}{ }^{2}\left(R^{2}+25 \omega^{2} L^{2}\right)+\ldots .\right]\right\}^{\frac{1}{2}}
\end{aligned}
$$

For brevity let

$$
\theta_{1}=\tan ^{-1} \frac{\omega L}{R}, \theta_{2}=\tan ^{-1} \frac{3 \omega L}{R}, \theta_{5}=\tan ^{-1} \frac{5 \omega L}{R} \ldots
$$

The active power in this circuit may then be expressed by

$$
\begin{aligned}
& P_{1}=\frac{I_{m}{ }^{2}}{2}\left[\left(R^{2}+\omega^{2} L^{2}\right)^{\frac{1}{2}} \cos \theta_{1}+k_{3}{ }^{2}\left(R^{2}+9 \omega^{2} L^{2}\right)^{\frac{1}{2}} \cos \theta_{3}\right. \\
& \left.+k_{5}{ }^{2}\left(R^{2}+25 \omega^{2} L^{2}\right)^{\frac{1}{2}} \cos \theta_{5}+\ldots .\right]
\end{aligned}
$$

If a hypothetical circuit whose current is $i_{2}$ (see eq 2) and voltage $e_{1}$ (see eq 8) is considered, its apparent power would be

$$
\begin{aligned}
& \left.U_{2}=E_{1} I_{2}=\frac{I_{m}{ }^{2}}{2 N}\left[\frac{1}{c_{1}{ }^{2}}+\frac{k_{3}{ }^{2}}{c_{3}{ }^{2}}+\frac{k_{5}{ }^{2}}{c_{5}{ }^{2}}+\ldots\right]\right]\left[R^{2}+\omega^{2} L^{2}\right. \\
& \left.\left.+k_{3}{ }^{2}\left(R^{2}+9 \omega^{2} L^{2}\right)+k_{5}{ }^{2}\left(R^{2}+25 \omega^{2} L^{2}\right)+\ldots .\right]\right]^{\frac{1}{2}}
\end{aligned}
$$

The active power in this hypothetical circuit would be

$$
\begin{aligned}
& P_{2}=\frac{I_{m}{ }^{2}}{2 N}\left[\frac{\left(R^{2}+\omega^{2} L^{2}\right)^{\frac{1}{2}}}{c_{1}} \cos \left(\theta_{1}-\beta_{1}\right)+k_{3}{ }^{2} \frac{\left(R^{2}+9 \omega^{2} L^{2}\right)^{\frac{1}{2}}}{c_{3}} \cos \left(\theta_{3}-\beta_{3}\right)\right. \\
& \left.+k_{5}{ }^{2} \frac{\left(R^{2}+25 \omega^{2} L^{2}\right)^{\frac{1}{2}}}{c_{5}} \cos \left(\theta_{5}-\beta_{5}\right)+\ldots\right]
\end{aligned}
$$


By referring to the first parts of eq 10 and 12 , and recalling that $I_{1}=N c_{e} I_{2}$, it can be seen that

$$
U_{1}=N c_{e} U_{2}
$$

The ratio of active power to apparent power can be represented by the cosine of some angle $\phi$, i. e.,

$$
\frac{P_{1}}{U_{1}}=\cos \phi_{1} \text { and } \frac{P_{2}}{U_{2}}=\cos \phi_{2}
$$

By substituting the values of $U_{1}$ and $U_{2}$ obtained from eq 15 in eq 14

$$
P_{1}=N c_{e} P_{2} \frac{\cos \phi_{1}}{\cos \phi_{2}}
$$

Equation 16 is a formula for the active power of the load circuit in terms of (a) the power $P_{2}$, measured with a wattmeter which has current $i_{2}$ flowing through its current circuit and voltage $e_{1}$ applied across its potential circuit; (b) the ratio, $N c_{e}$, of the current transformer used in transforming $i_{1}$ to $i_{2}$; and (c) another factor, $\cos \phi_{1} / \cos \phi_{2}$, involving the phase angle of this current transformer and the power factor of the load circuit. Under sinusoidal conditions this last factor would be $1 /(\cos \beta+\tan \gamma \sin \beta)$, where $\beta$ is the phase angle of the current transformer and $\cos \gamma$ is the power factor of the load circuit. The following arbitrary equation

$$
\frac{\cos \phi_{1}}{\cos \phi_{2}}=\frac{1}{\cos \beta+\tan \gamma \sin \beta}
$$

can be set up and if a value of $\beta$ in terms of $\beta_{1}, \beta_{2}, \beta_{3}, \cdots c_{1}, c_{3}, c_{5}, \cdots$ can be found which satisfies this equation, then this value of $\beta$ can be used as the definition of the phase angle of a current transformer under nonsinusoidal conditions. The main advantage of this definition is that the phase angle so defined can be correctly used in the customary formula for power when a current transformer is used in conjunction with a wattmeter for power measurements.

The first step in solving eq 17 is to obtain a value for $\cos \phi_{2} / \cos \phi_{1}$ by referring to eq 15 and substituting the values of $U_{1}, P_{1}, U_{2}$, and $P_{2}$ given in eq 10 to 13 . After simplifying, this relation becomes

$$
\begin{aligned}
& \frac{\cos \phi_{2}}{\cos \phi_{1}}=\frac{\left(\cos \beta_{1}+k_{3}{ }^{2} \frac{c_{1}}{c_{3}} \cos \beta_{3}+k_{5}{ }^{2} \frac{c_{1}}{c_{5}} \cos \beta_{5}+\cdots\right)+}{\left[( 1 + k _ { 3 } { } ^ { 2 } + k _ { 5 } { } ^ { 2 } + \cdots \cdot ) ^ { \frac { 1 } { 2 } } \left(1+k_{3}{ }^{2} \frac{c_{1}{ }^{2}}{c_{3}{ }^{2}}+\right.\right.} \\
& \frac{\tan \theta_{1}\left(\sin \beta_{1}+3 k_{3}{ }^{2} \frac{c_{1}}{c_{3}} \sin \beta_{3}+5 k_{5}{ }^{2} \frac{c_{1}}{c_{5}} \sin \beta_{5}+\cdots\right)}{\left.\left.\left.k_{5}{ }^{2} \frac{c_{1}{ }^{2}}{c_{5}{ }^{2}}+\cdots\right)\right)^{\frac{1}{2}}\right]}
\end{aligned}
$$

The denominator of the right-hand side of eq 18 may be replaced by the following series, which is a sufficiently close approximation if $k_{n} \leqq 0.2$

$$
1+k_{3}{ }^{2} \frac{c_{1}}{c_{3}}+k_{5}{ }^{2} \frac{c_{1}}{c_{5}}+\cdots
$$


Making this substitution and performing the indicated division gives a value for $\cos \phi_{2} / \cos \phi_{1}$, which, when placed in eq 17 , gives

$$
\begin{aligned}
& \cos \beta+\tan \gamma \sin \beta=\cos \beta_{1}+\frac{k_{3}{ }^{2} \frac{c_{1}}{c_{3}}\left(\cos \beta_{3}-\cos \beta_{1}\right)+k_{5}{ }^{2} \frac{c_{1}}{c_{5}}\left(\cos \beta_{5}-\cos \beta_{1}\right)+\cdots}{1+k_{3}{ }^{2} \frac{c_{1}}{c_{3}}+k_{5}{ }^{2} \frac{c_{1}}{c_{5}}+\cdots} \\
& +\tan \theta_{1}\left[\sin \beta_{1}+\frac{k_{3}{ }^{2} \frac{c_{1}}{c_{3}}\left(3 \sin \beta_{3}-\sin \beta_{1}\right)+k_{5}{ }^{2} \frac{c_{1}}{c_{5}}\left(5 \sin \beta_{5}-\sin \beta_{1}\right)+\cdots}{1+k_{3}{ }^{2} \frac{c_{1}}{c_{3}}+k_{5}{ }^{2} \frac{c_{1}}{c_{5}}+\cdots}\right]
\end{aligned}
$$

Under sinusoidal conditions, $\gamma$ is defined as the angle between the voltage and the current. By defining $\gamma$ under nonsinusoidal conditions as the angle between the fundamental components of the voltage and the current, $\tan \gamma$ is made equal to $\tan \theta_{1}$.

For nearly all commercial current transformers $\beta_{n} \leqq 120$ minutes. For such small angles $\sin \beta_{n}$ can be replaced by $\beta_{n}$ and $\cos \beta_{n}$ by $1-\beta_{n}{ }^{2} / 2$ and eq 19 becomes

$$
\begin{aligned}
& 1-\frac{\beta^{2}}{2}+\tan \theta_{1}(\beta)=1-\frac{\beta_{1}{ }^{2}}{2}+\frac{k_{3}{ }^{2} \frac{c_{1}}{c_{3}}\left(\frac{\beta_{1}{ }^{2}-\beta_{3}{ }^{2}}{2}\right)+k_{5}{ }^{2} \frac{c_{1}}{c_{5}}\left(\frac{\beta_{1}{ }^{2}-\beta_{5}{ }^{2}}{2}\right)+\ldots}{1+k_{3}{ }^{2} \frac{c_{1}}{c_{3}}+k_{5}{ }^{2} \frac{{ }^{2}}{c_{5}} \ldots} \\
& +\tan \theta_{1}\left[\beta_{1}+\frac{k_{3}{ }^{2} \frac{c_{1}}{c_{3}}\left(3 \beta_{3}-\beta_{1}\right)+k_{5}{ }^{2} \frac{c_{1}}{c_{5}}\left(5 \beta_{5}-\beta_{1}\right)+\ldots}{1+k_{3} \frac{{ }^{2} \frac{c_{1}}{c_{3}}+k_{5}{ }^{2}}{c_{5}}+\ldots}\right]
\end{aligned}
$$

For nearly all commercial current transformers $\beta-\beta_{1} \leqq 15$ minutes and $\beta_{1}-\beta_{n} \leqq 60$ minutes, and since $k_{n} \leqq 0.2$ the following maximum values can be set for certain terms in the above equation:

$$
\begin{gathered}
\frac{\beta^{2}-\beta_{1}{ }^{2}}{2} \leqq 0.00012 \\
\frac{k_{3}{ }^{2} \frac{c^{1}}{c_{3}}\left(\frac{\beta_{1}{ }^{2}-\beta_{3}{ }^{2}}{2}\right)+k_{5}{ }^{2} \frac{c_{1}}{c_{5}}\left(\frac{\beta_{1}{ }^{2}-\beta_{5}{ }^{2}}{2}\right)+\ldots}{1+k_{3}{ }^{2} \frac{c_{1}}{c_{3}}+k_{5}{ }^{2} \frac{c_{1}}{c_{5}}+\ldots} \leqq 0.00006
\end{gathered}
$$

Equation 20 now becomes

$$
\begin{aligned}
& 1+\tan \theta_{1}(\beta)=1+\tan \theta_{1}\left[\beta_{1}\right.
\end{aligned}
$$

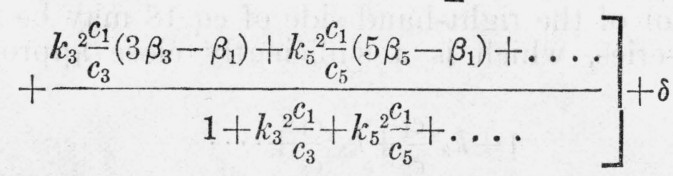


where $\delta \leqq 0.00018$, i. e., the maximum error introduced by neglecting $\delta$ is less than 0.02 percent. For nearly all measurements of power or energy, this error is negligible and eq 21 becomes

$$
\beta=\beta_{1}+\frac{k_{3}{ }^{2} \frac{c_{1}}{c_{3}}\left(3 \beta_{3}-\beta_{1}\right)+k_{5}{ }^{2} \frac{c_{1}}{c_{5}}\left(5 \beta_{5}-\beta_{1}\right)+\ldots}{1+k_{3}{ }^{2} \frac{c_{1}}{c_{3}}+k_{5}{ }^{2} \frac{c_{1}}{c_{5}}+\ldots}
$$

Equation 22 is a definition of the phase angle of a current transformer for nonsinusoidal currents in terms of the ratios and the phase angles of transformation for the fundamental and for the harmonic components of the current being measured. The approximations used in deriving eq 22 hold true for all of the transformers tested during this investigation except two which were intentionally of poor design. For these two transformers the maximum error introduced by the approximations was less than 0.04 percent.

\section{APPARATUS AND PROCEDURE}

The apparatus ${ }^{3}$ regularly employed at the National Bureau of Standards in testing current transformers was used in the experimental work of this investigation. For the usual measurements with this apparatus the detector is a single vibration galvanometer which is sensitive only to the fundamental frequency of the current being used. During the present investigation the current wave form was varied from the sinusoidal by the introduction of a third harmonic. It was therefore necessary to provide an alternate detector sensitive to the third harmonic. This was the only addition to the apparatus regularly employed in testing current transformers.

The source of the current used in the experimental work consisted of two revolving-field alternating-current generators mounted on the same shaft with a direct-current motor which was supplied from a storage battery. The rated frequencies of the generators were 60 and 180 cycles per second, respectively. The terminal voltages of the 60 -cycle and the 180-cycle generators contained not more than 2 and 5 percent, respectively, of any harmonic under the conditions of load used for this investigation. The stator of the 180-cycle generator was mounted in such a way that it could be rotated with respect to the stator of the 60-cycle generator through 180 electrical degrees of the 60-cycle generator.

Three different wave forms of primary current were used in the experimental work: (a) sinusoidal; (b) peaked, consisting of the fundamental plus a 20 percent third harmonic, the positive crest of the fundamental coinciding with a positive crest of the harmonic; (c) flat top, consisting of the fundamental plus a 20 percent third harmonic, the positive crest of the fundamental coinciding with a negative crest of the harmonic (see fig. 1 for sketches of these three wave forms). The sinusoidal wave form was obtained by using the 60 -cycle generator only. The nonsinusoidal wave forms were obtained by connecting the stators of the two generators in series. The amplitude and phase of the third harmonic were adjusted by varying the field excita-

\footnotetext{
${ }^{3}$ For a complete description of this apparatus, see BS J. Research 11, 93 (1933) RP580.
} $19521-37-3$ 
tion of the 180-cycle generator and the angular position of its stator. The amplitude of the third harmonic was measured with a wattmeter whose current coils were connected in the secondary circuit of the transformer being tested and whose voltage circuit was connected to an independent 180 -cycle source whose phase relation with respect to

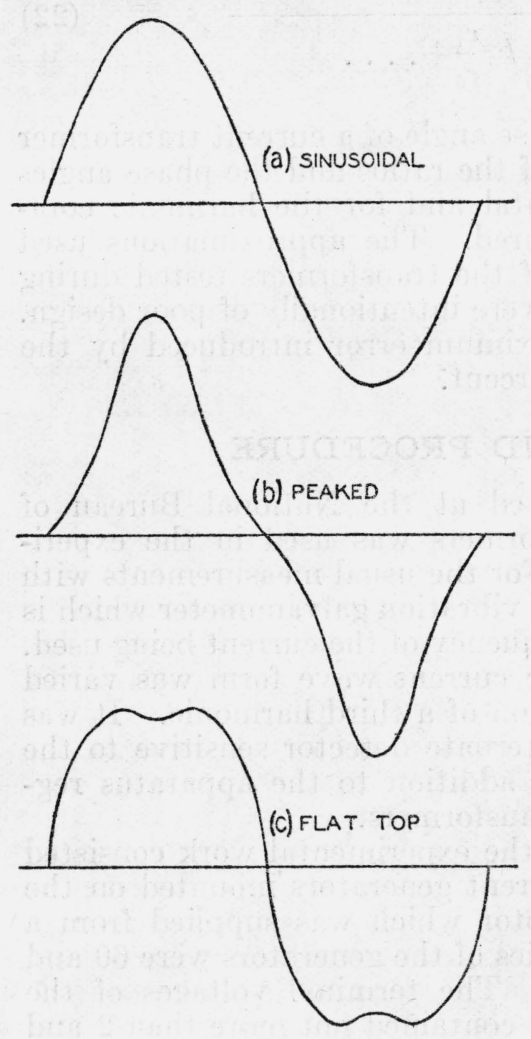

FIgURe 1.-Drawings of the three wave forms used in the experimental work.

Waves (b) and (c) each consisted of the fundamental, plus a 20 -percent third harmonic. the test supply could be controlled.

This phase relation was adjusted to give maximum deflection on the wattmeter. By using this maximum value of watts and the known value of 180 -cycle voltage impressed on the voltage circuit of the wattmeter, the rms value of the 180-cycle component of current in the secondary circuit of the current transformer was computed. The phase relation between the third harmonic and the fundamental was observed by viewing the wave form of the secondary current on a galvanometer-type oscillograph.

The procedure in determining the effect of changing the current wave form upon the ratios and the phase angles of current transformers was first to measure the ratio and the phase angle of the transformer in the customary manner at six values of secondary current, using a current of sinusoidal wave form. Then another set of measurements was made with all conditions duplicated except that a current of peaked wave form was used. In this second set of measurements two values of ratio and phase angle were obtained for each setting of the secondary current, one being the ratio and phase angle between the fundamental components of the primary current and the secondary current and the other between the third harmonic components. These values were obtained using the 60 -cycle and the 180 -cycle vibration galvanometers, respectively. A third set of measurements was next made in the same manner as the second set except that a current of flat-top wave form was used. The ratios and the phase angles of the current transformer for nonsinusoidal currents were computed from the ratios and phase angles for the fundamental and for the third harmonic by means of eq 7 and 22, respectively. This procedure was duplicated for each transformer tested and for some transformers it was repeated using a different arrangement of primary leads.

Since the Silsbee current-transformer testing set is a well-known and widely used apparatus for comparing the ratios and phase angles of two transformers of the same nominal ratio, it was deemed ad- 
visable to check the performance of one of these sets, using a nonsinusoidal current. Two transformers of the same nominal ratio were chosen and their ratios and phase angles were first measured by the absolute method as described above for the three different wave forms. Then the ratios and phase angles of these transformers were compared by means of a Silsbee testing set, using in succession currents of each of the three wave forms used in the absolute measurements. The results of this test indicate how nearly sinusoidal the current should be in order to obtain a given accuracy of measurement when using one of these testing sets.

\section{RESULTS AND CONCLUSIONS}

Seven current transformers, having various core shapes and arrangements of windings and primary current ratings up to 2,000 amperes, were tested according to the procedure just outlined. The results on a few of these transformers were chosen as being sufficient to indicate

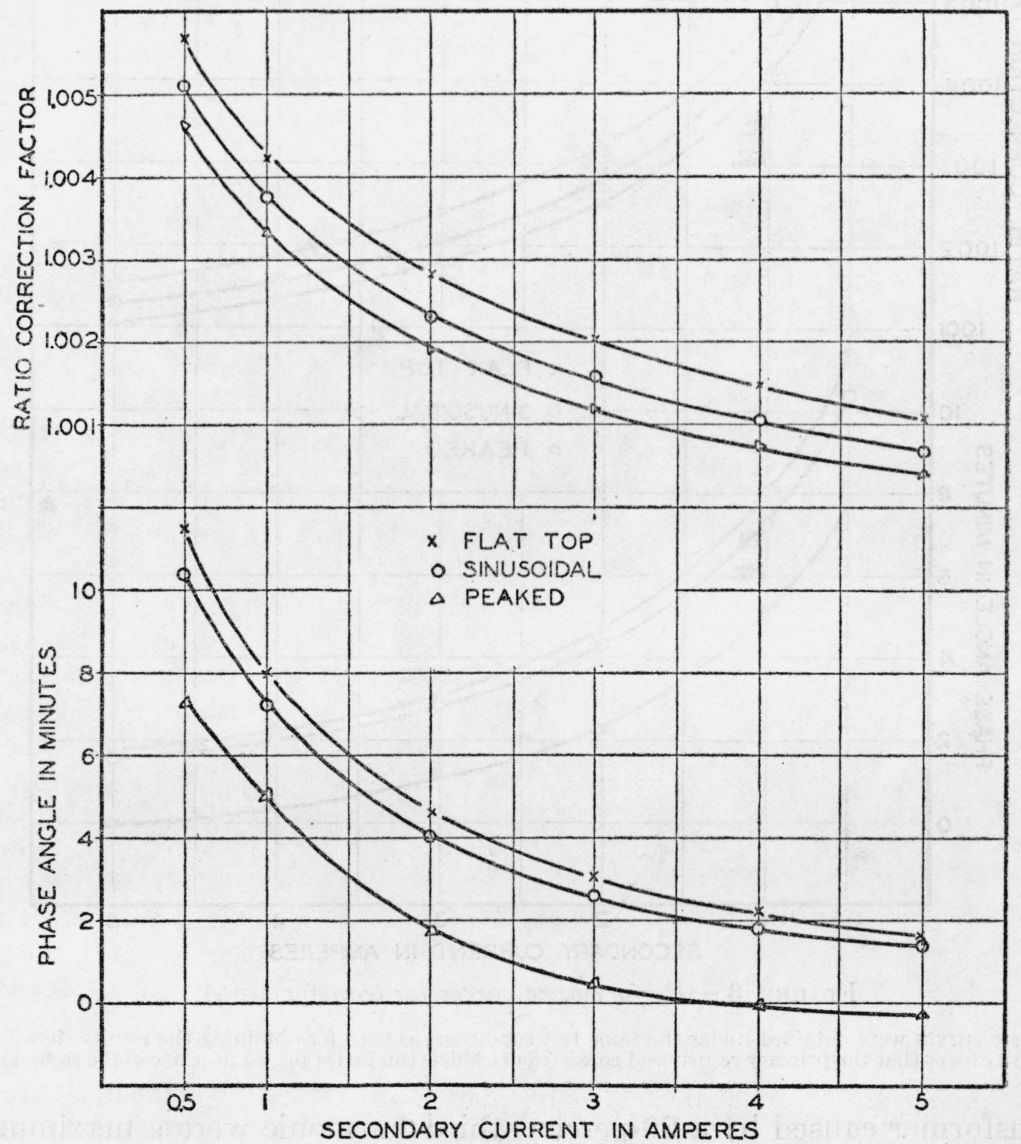

Figure 2.-Performance curves for transformer $A$.

Nominal ratio 2000:5. Burden $0.4 \mathrm{ohm}$ and 310 microhenries. Frequency 60 cycles per second. Rectangular-shaped core with two secondary coils of 200 turns each on opposite legs of the core. Primary circuit consisting of a single straight conductor through the center of the core window and four return leads uniformly spaced around the central conductor. 
the magnitude of changes in ratio and phase angle when the wave form departs from the sinusoidal. These results are shown by the curves of figures 2 to 5 .

The results on transformer $A$, shown in figure 2 , were chosen as being typical of commercial current transformers of good design for large currents. The distortion of a sine wave caused by the introduction of a 20-percent third harmonic can easily be detected by visual observation of the wave on an oscillograph. With distortions of this magnitude, it is even possible to estimate the magnitude and phase relation of the harmonic by visual observation (see fig. 1). However, as shown by the curves of figure 2 , the changes in performance of this

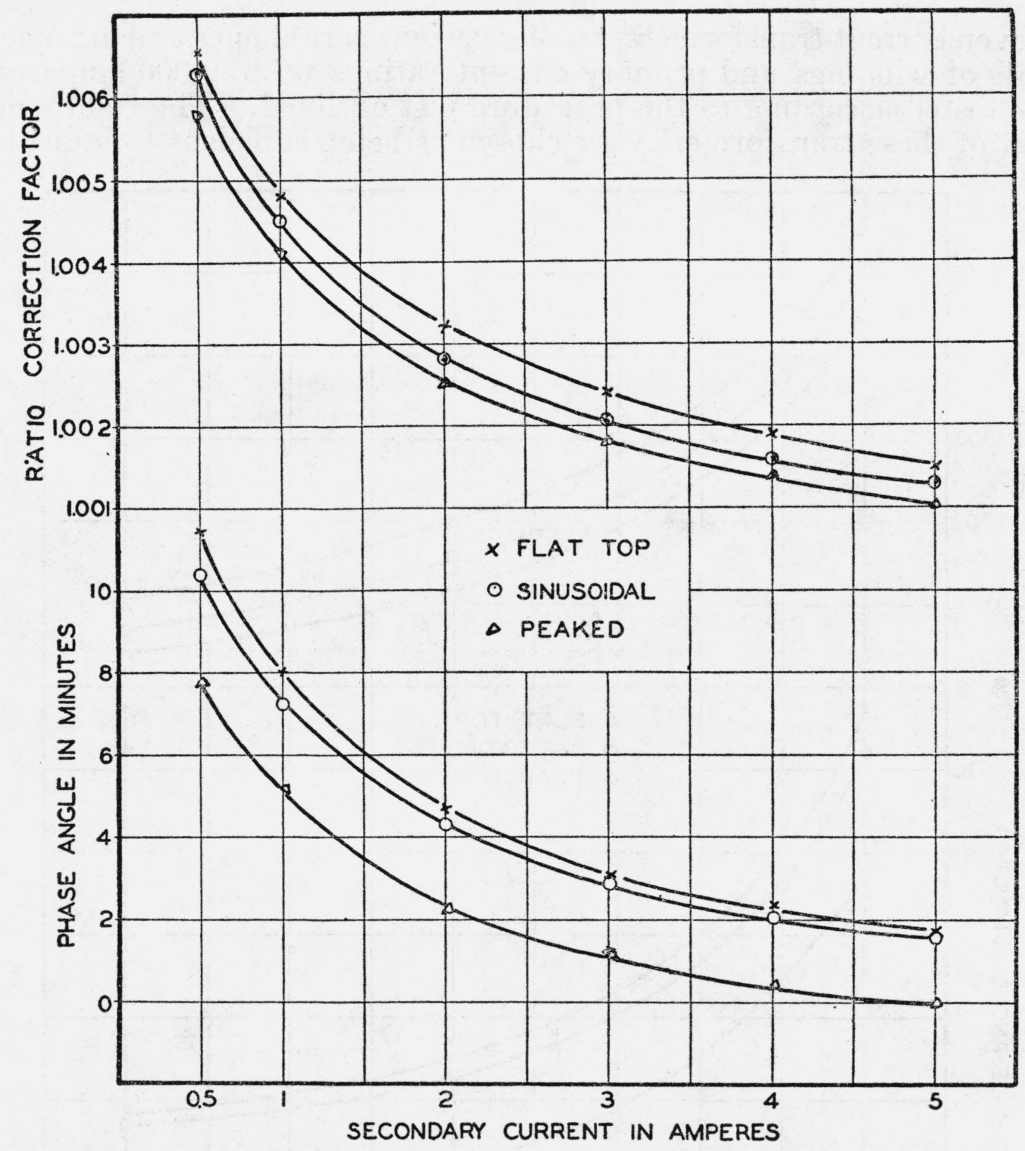

Figure 3.-Performance curves for transformer $A$.

These curves were obtained under the same test conditions as used for obtaining the results shown in figure 2 except that the primary return lead consisted of a single conductor placed near one of the secondary coils.

transformer caused by a 20-percent third harmonic were a maximum of only 0.05 percent in ratio and 2 minutes in phase angle. Both the ratio and the phase angle are greater for a flat-top wave form than for a sinusoidal wave form and less for a peaked wave form. These results may be accounted for by the following reasoning. The wave 
form of the primary current and the secondary current of a welldesigned current transformer is essentially the same as the wave form of the voltage induced in the windings by the core flux. If this wave form is changed from sinusoidal to peaked, keeping the effective value constant, the average value will be less. A lower value of average induced voltage requires a lower maximum value of core flux and less exciting ampere-turns, thus decreasing the ratio and the phase angle. If the wave form is changed from sinusoidal to flat top the effect will be the opposite.

The results shown in figure 3 were obtained from the same transformer and under the same test conditions as the results shown in figure 2, except that the primary return lead was arranged in such a way as to produce a large value of primary leakage flux in one leg of the core. Under these conditions the performance ${ }^{4}$ of a current transformer involves a number of factors which are not readily predictable, and changing the wave form further complicates the predictability of these factors. However, by comparing figures 2 and 3 it can be seen that the changes in performance with changes in wave form are only very slightly different for these two widely different arrangements of primary return lead, the change in ratio due to the change in arrangement of primary return lead being greater than that due to the change in wave form.

Transformers $B$ and $C$ were intentionally constructed as examples of very poor design. Transformer $B$ had a very low value of secondary ampere-turns and transformer $C$ had an extremely nonuniform distribution of primary turns and secondary turns. The results of the tests on these transformers (shown in fig. 4 and 5 which are plotted to a much less open scale than fig. 2 and 3) indicate that, although their general performance characteristics are very poor, the changes in performance with changes in wave form are not excessive, being a maximum of 0.2 percent in ratio and 20 minutes in phase angle. Because of the high values of leakage flux in transformer $C$, the flux density in certain sections of its core is abnormally high. Since the wave form of leakage flux is the same as the wave form of the current producing it, for peaked wave forms of current, the values of core flux density go beyond the point of maximum permeability for secondary currents above 3 amperes, thus causing the ratio correction factor to increase, as shown in figure 5. Transformers of such poor design as $B$ and $C$ would not be used in practice either as standards or for measurements of power or energy.

In all of the results shown in figures 2 to 5 , effective values of ratio correction factor and phase angle were used, i. e., the values were computed from measurements on the fundamental and third-harmonic component by use of eq 7 and 22. For all of the transformers tested except $B$ and $C$, the effective values of ratio and phase angle differed from the values obtained using fundamental components only, by a maximum of 0.01 percent in ratio and 1.5 minutes in phase angle. For transformers $B$ and $C$ these differences were a maximum of 0.13 percent in ratio and 12 minutes in phase angle.

The ratios and phase angles of two commercial current transformers (nominal ratio 100:5) of good design were compared by means of a Silsbee current-transformer testing set, using each of the three different wave forms of primary current used in the previous tests. It was

\footnotetext{
'See pages 370 and 371 of J. Research NBS 14 (1935) RP775.
} 
found that the results using the testing set (to the limits of its accuracy) agreed with the results obtained by the absolute method.

The detector of a Silsbee testing set consists of a separately excited galvanometer. Its moving coil is connected in the detector circuit. Its fixed coil is supplied by an external circuit whose frequency and (for this investigation) wave form are the same as those of the supply voltage being used for the transformer testing. A phase-shifting

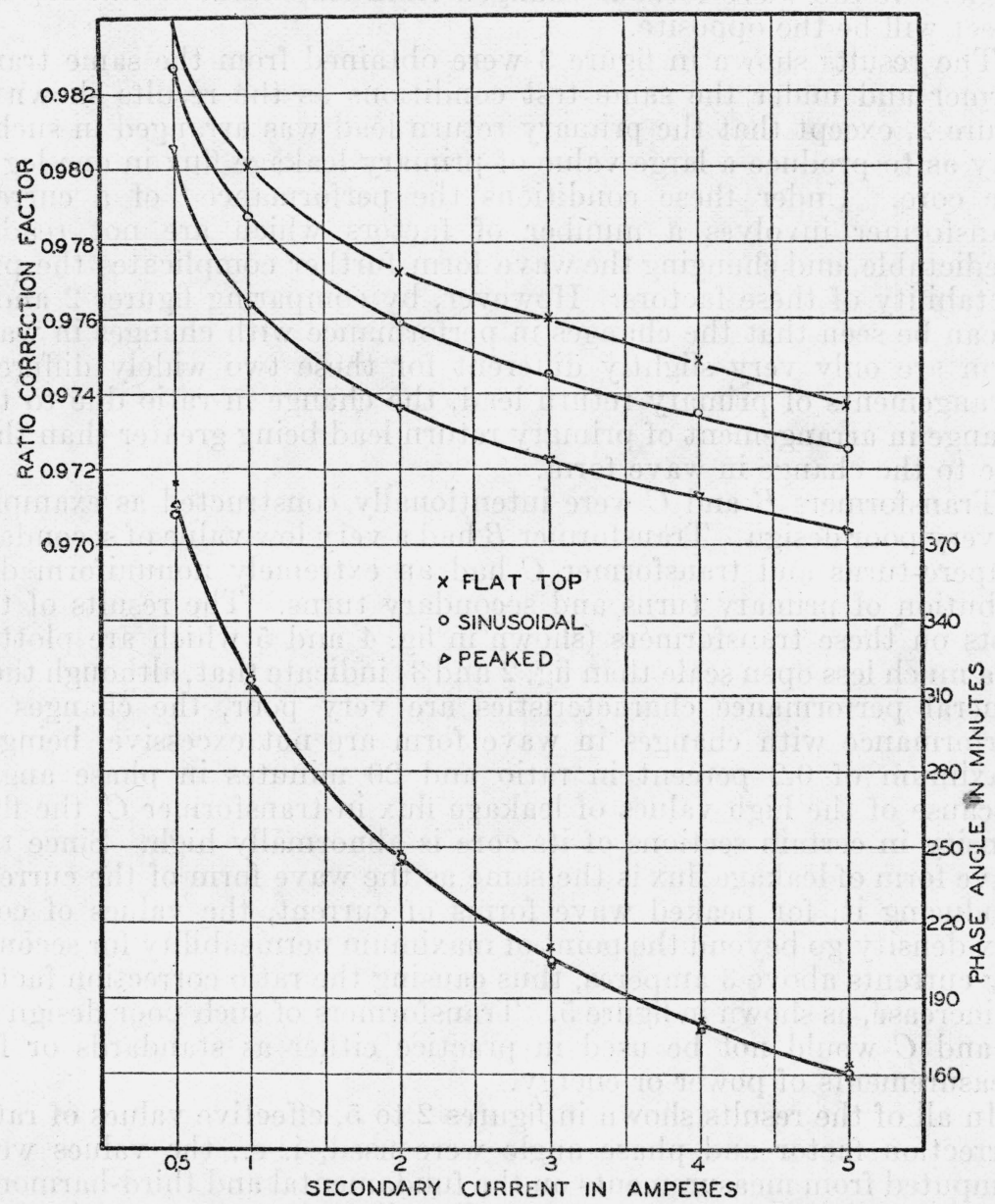

Figure 4.-Performance curves for transformer $B$.

Nominal ratio 50:5. Burden $0.16 \mathrm{ohm}$. Rectangular-shaped core with 38 secondary turns uniformly distributed around it. Four primary turns, one around each leg of the core.

transformer in the supply circuit for the galvanometer excitation serves as a means of adjusting the phase relation between the exciting: current and the primary current of the transformers being tested. $\AA$ balance is obtained by adjusting alternately the ratio and the phaseangle dials of the testing set while the phase-shifting transformer is set for each alternate adjustment to give sensitivity for the quantity indicated by the dial being adjusted. 
A balance obtained by means of such a detector may reasonably be expected to depend not only upon the relation between the fundamental components of the currents being compared but also, at least to a slight extent, upon any harmonic components present in these currents. Since the phase relation between the fundamental and the harmonic components may be quite different for the galvanometer exciting current than for the current being detected, the extent of the

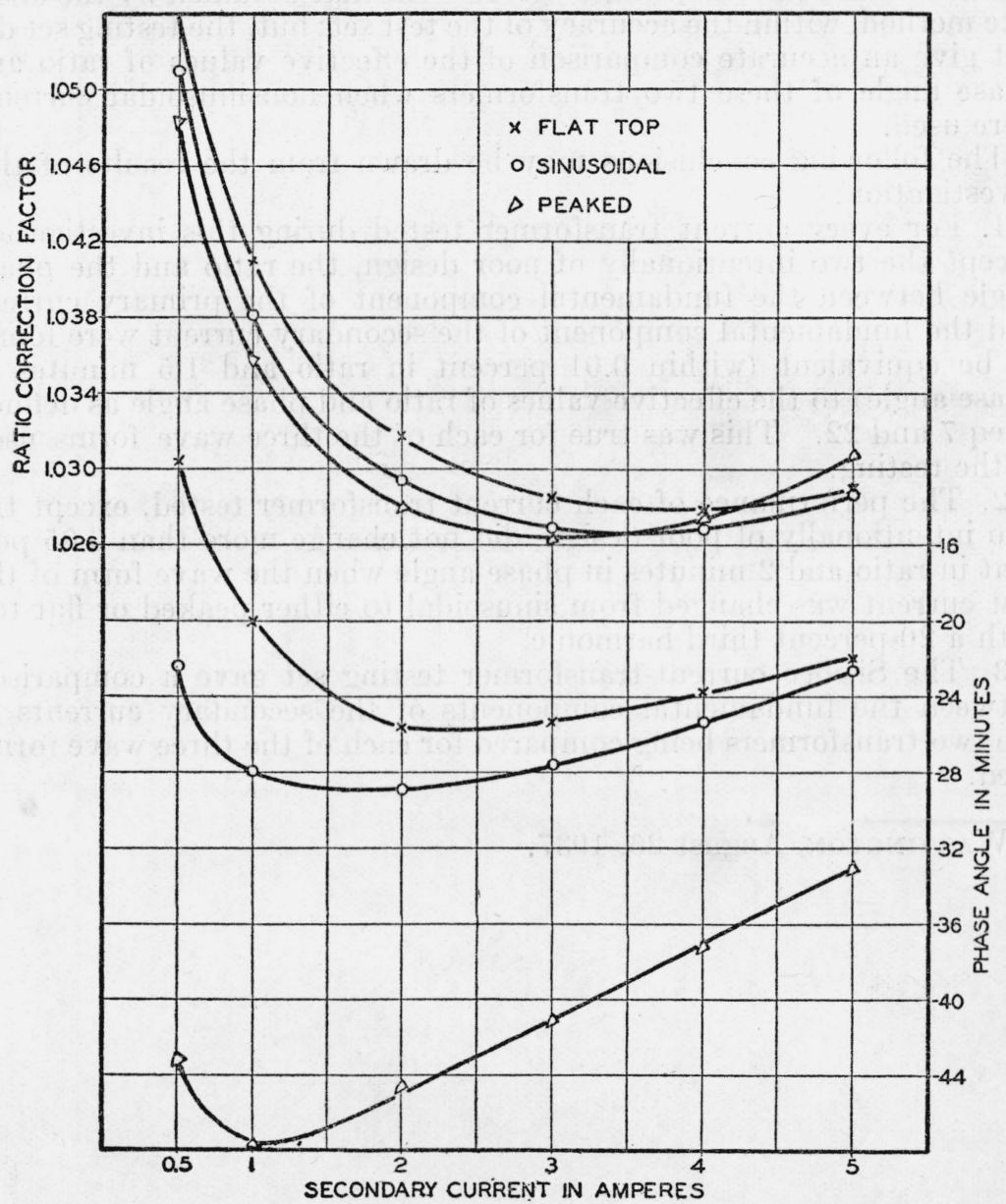

FIGURE 5.-Performance curves for transformer $C$.

Nominal ratio $100: 5$. Burden $0.4 \mathrm{ohm}$ and 310 microhenries. Rectangular-shaped core with one secondary coil of 200 turns around one leg of the core. Primary circuit consisted of 10 turns on opposite leg of the core.

effect of the harmonic components on the detector balance cannot readily be derived. It was decided that the magnitude of such an effect could be measured experimentally more readily than it could be calculated theoretically.

For the two commercial current transformers of good design whose performance characteristics were compared by the use of a Silsbee testing set, the differences between the effective values of ratio and 
phase angle and the values obtained using fundamental components only were too small to discriminate which of the two types of values was being indicated by the testing set.

In order to determine this, transformer $C$, the one of poor design referred to above, was compared with a transformer of good design by use of the testing set. The results showed that the testing set gave a comparison of the ratios and phase angles of the fundamental components of the currents, which agreed with that obtained by the absolute method, within the accuracy of the test set; but, the testing set did not give an accurate comparison of the effective values of ratio and phase angle of these two transformers when nonsinusoidal currents were used.

The following conclusions may be drawn from the results of this investigation:

1. For every current transformer tested during this investigation except the two intentionally of poor design, the ratio and the phase angle between the fundamental component of the primary current and the fundamental component of the secondary current were found to be equivalent (within 0.01 percent in ratio and 1.5 minutes in phase angle) to the effective values of ratio and phase angle as defined in eq 7 and 22. This was true for each of the three wave forms used in the testing.

2 . The performance of each current transformer tested, except the two intentionally of poor design, did not change more than 0.05 percent in ratio and 2 minutes in phase angle when the wave form of the test current was changed from sinusoidal to either peaked or flat top with a 20-percent third harmonic.

3 . The Silsbee current-transformer testing set gave a comparison between the fundamental components of the secondary currents of the two transformers being compared for each of the three wave forms used.

Washington, August 26, 1937. 